\title{
IDENTIFICATION ET CLONAGE MOLÉCULAIRE DE GENES HUMAINS DE LA RÉPARATION PAR EXCISION
}

\author{
Mauro MEZZINA \\ Groupe de Laboratoires de l'I.R.S.C. \\ UPR 42 CNRS, BP. $n^{\circ} 8$, \\ 94802 Villejuif
}

Le mantien de l'intégrité du matériel génétique est une conditiom essentielle pour la vie de la cellule et ceci est assuré par les processus de réparation de l'ADN. Si une mutation dans un gène de réparation perturbe le fonctionnement de ce processus, la cellule n'est plus viable, ou alors certains mécanismes cellulaires sont induits en engendrant des changements dans le programme génétique qui peuvent aboutir à la transformation cellulaire. Les rares maladies génétiques humaines de la réparation de I'ADN, comme le xeroderma pigmentosum (XP), l'anémie de Fanconi (AF), l'ataxie télangectasie (AT) ou la syndrome de Bloom (BS) sont donc caractérisées par une instabilité génétique et une forte prédisposition au cancer. Néammoins, si l'on veut étudier les différents gènes impliqués dans le processus de réparation de l'ADN chez l'homme, ces maladies présentent un système génétique modèle (1).

La maladie XP est la mieux connue sur le plan génétique et biochimique. Les malades XP présentent une sensibilité à l'irradiation ultraviolette à $254 \mathrm{~nm}$ et à d'autres agents cancérigènes et il a été démontré que les cellules de ces malades présentent un défaut dans la réparation de lésions sur l'ADN induites par un tel traitement. De plus, ces cellules ont un taux très élévé de mutabilité et $100 \%$ des malades XP présentent des cancers cutanés dans les parties du corps éxposées au soleil (2). Cette maladie est donc un modèle bielogique pour étudier les liaisons cntre la réparation, la mutagénèse et la cancérogénèse chez l'homme.

Deux autres maladies génétiques humaines présentent un phénotype de sensibilité aux UV et de défaut de réparation similaire, la trichothiodystrophie (TTD) et le syndrome de Cockayne (CS). Toutefois, aucun de ces malades ne développe de cancers cutanés. L'etude des gènes de la réparation par excision peut donc nous aider à mieux comprendre le paradoxe biologique représenté par ces trois maladies.

Lanalyse génétique de ces trois maladies a mis en evidence 7 groupes de complémentation pour XP (groupes A-G plus un huitiéme groupe de XP "variant"), et 3 groupes pour CS (A-C). Pour les malades TTD photosensibles, la majorité appartient au même groupe de complémentation des cellules XP$D$, et trois autres malades (TTD1BR et TTD4VI et TTD6VI), récemment caractcrisés, appartiendraient à deux nouveaux groupes de complémentation $(3,4)$. Récemment, plusieurs individus XP appartenant aux groupes de complémentation $B$, $D$ et $G$ manifestant aussi la pathologic typique du syndrome de Cockayne ont été decrit (2). Il semble donc que la réparation de l'ADN soit un processus très complexe impliquant de nombreux gènes. Il est possible que certaines mutations responsables du phénotype de radiosensibilité ainsi que de différentes anomalies dans le processus de la réparation de l'ADN dans les cellules XP, CS et TTD puissent être localisées dans des gènes communs (comme dans le cas de malades XP-D et de la majorité des TTD photosensibles) ou proches les uns des autres. Ceci donnerait lieu au même phénotype cellulaire (défaut de réparation de l'ADN) mais à des manifestations pathologiques différentes chez les malades. 
Depuis le développement des techniques de clonage des gènes, la compréhension de la réparation de I'ADN a beaucoup progressé. Notamment, plusieurs gènes ont été identifiés et clonés; pour certains d'entre eux, l'homologie avec les gènes de réparation dans les organismes inférieurs nous donne une idée de leur fonction. Notamment, le gène XP-AC (XP-A correcting) complémentant les cellules XP-A (5), ERCC3 (excision repair cross complementation) complémentant XP-B/CS (6), XP-CC complémentant XP-C (7), ERCC2 complémentant XP-D et TTD/XP-D (8) et ERCC6 complémentant CS-B (9). D'aprés leurs séquences, ERCC2 et ERCC3 contiennent des domains fonctionnels d'hélicases, et leurs produits agiraient probablement en synérgie sur l'ADN endommagé (6).

\section{GENES HUMAINS DE LA REPARATION PAR EXCISION}

$\begin{array}{lllll}\begin{array}{l}\text { groupes de } \\ \text { complémentation }\end{array} & \text { gène } & \begin{array}{l}\text { nombre de } \\ \text { AA présumé }\end{array} & \begin{array}{l}\text { localisation } \\ \text { chromosomique }\end{array} & \begin{array}{l}\text { homologu } \\ \text { chez la } \\ \text { levure }\end{array} \\ \text { XP-A } & & & & \text { RAD14 } \\ \text { XP-B } & \text { XP-AC } & 273 & 9 \mathrm{q} 34 & \text { RAD25 } \\ \text { XP-C } & \text { ERCC3 } & 782 & 2 \mathrm{q} 21 & \text { RAD4 } \\ \text { XP-D } & \text { XP-CC } & 823 & & \text { RAD3 } \\ \text { XP-E } & \text { ERCC2 } & 760 & 19 \mathrm{q} 13 & \\ \text { XP-F } & & & 15 & \\ \text { XP-G } & & & & \\ \text { CS-A } & & & 10 q 11 & \\ \text { CS-B } & \text { ERCC6 } & 1493 & & \text { RAD10 } \\ \text { TTD/XP-D } & \text { ERCC2 } & & & \\ \text { TTD1BR } & & & & \\ \text { TTD6VI } & \text { ERCC1 } & 297 & 19 \mathrm{q} 13 & \text { RAD6 } \\ & \text { ERCC4 } & & 13 & \text { RAD23 }\end{array}$

Les stratégies adoptées pour identifier et cloner ces gènes sont différentes. Ici seront décrites et discutées les deux principales.

L'une consiste à transfecter certaines lignées de cellules de rongeurs (CHO) présentant un phénotype de sensibilité aux UV avec de l'ADN génomique humain sauvage couplé à un marqueur de sélection comme le gène neo, qui confère une resistance à l'antibiotique G418 aux cellules ayant intégré l'ADN exogène. Après une première sélection en G418, les cellules sont sélectionnées pour leur resistance aux UV. L'ADN de clones de cellules de rongeurs résistants aux UV est ensuite fragmenté et retransfecté dans les cellules CHO. L'ADN des clones resistants aux UV est ensuite cloné dans une banque de cosmides ou de phages. Les cosmides ou phages contenant de l'ADN humain seront ensuites criblés par hybridation préferentielle (l'ADN humain ne s'hybridant pas avec celui du rongeur). Cette technique permet donc d'identifier directement le géne de réparation. En utilisant cette technique, il a été possible d'isoler et de cloner les gènes ERCC (11). Une variation de cette méthode consiste à utiliser les cellules humaines comme cellules receveuses et l'ADN génomique de rongeurs dans les transfections. 
On obtient ainsi des séquences d'ADN de rongeur capables de corriger la photosensibilité des cellules humaines. Avec ces séquences on peut ensuite cribler des banques d'ADN complémentaire (ADNc) humain et identifier le gène homologue chez l'homme. En utilisant cette méthode il a été possible d'identifier l' ADNc XP-AC (5).

Une strategie alternative consiste à utiliser des vecteurs d'expression EBV (vecteurs navettes basés sur le virus Epstein Barr) contenant une banque d'ADNc humain. L'avantage de l'utilisation de cette stratégie reside dans le fait que ces vecteurs se répliquent et expriment les séquences d'ADN clonées, en restant des unités épisomales. La banque d'ADNc peut donc être introduite dans les cellules par transfection ou électroporation et les cellules exprimant de façon stable le vecteur peuvent être sélectionnées en utilisant du milieu contenant de l'hygromycine. Cette approche permet d'obtenir des efficacités très élévées d'expression stable du vecteur dans la ccllule, car l'étape d'intégration dans I'ADN cellulaire n'est plus limitante. En effet, la limite de la première méthode est que l'expression de sćquences génomiques exogènes nécessite l'intégration dans l'ADN cellulaire, ce qui est un évènement de très faible fréquence. Il est donc possible d'obtenir rapidement avec les vecteurs EBV, au moins 100.000 clones (100.000 est le nombre estimé des gènes existant chez l'homme) de cellules préselectionnées à l'hygromycine et de sélectionner les cellules ayant acquis la complémentation phénotypique en irradiant directement les clones résistants. Un autre avantage présenté par cette approche est la possibilité d'extraire l'ADN du plasmide, à partir des clones potentiellement complémentants, par la méthode de Hirt et de le réintroduire dans la bactérie $E$. coli. L'ADN plasmidique amplifié dans la bactérie peut être ensuite vérifié pour sa propriété de complémentation cn le retransfectant dans les celiules.

Lorsque les séquences d'ADN complémentant ie phénotype de scinsibilité aux UV seront identifiées, la caractérisation des gènes en question pourra s'effectuer en utilisant les techniques courantes de la biologie molćculairc des gènes clonés.

La derniêre strategie s'est révélée celle donnant le plus de résultats, notamment, les gène XP-CC et FA-CC, ce dernier complémentant les cellules de l'Anémie de Fanconi groupe $C$, ont pu être identifiés et clonés (10).

\section{REFERENCES}

1. Hoeijmakers, J.H.J. and Bootsma, D. (1992) DNA repair: two pieces of the puzzle Nature Genetics 1, 313-314

2. Cleaver, J.E. and Kracmer, K.H. (1992) Xeroderma Pigmentosum and Cockayne Syndrome in Metabolic Basis of Inherited Disease in press

3. Stefanini, M., Lagomarsino, P., Gillani, S., Nardo, T., Botta, E., Kleijer, W., Lehmann, A. and Sarasin, A. (1992) Genetic heterogeneity of the excision repair defect associated with trichothiodystrophy. Cancer Research submitted

4. Stefanini, M., Vermeulen, W., Giliani, S., Nardo, T., Mezzina, M., Sarasin, A., Harper, J.I., Arlett, C.F., Hoeijmakers, J.H.J. and Lehmann, A.R. (1992) A newnucleotide excision repair gene associated with the genetic disorder trichothiuodystrophy. Nature Genetics, submitted.

5. Tanaka, K., Satokata, I., Ogita, Z, Uchida, T. and Okada, Y. (1989) Molecular cloning of a mouse DNA repair gene that complements the defect of group-A xeroderma pigmentosum. Proc. Natl. Acad. Sci. USA, 86, 5512-5516.

6. Weeda, G. van Ham, R.C.A., Vermeulen, W., Bootsma, D., van der Heb, A.J. and Hoeijmaker, J.H.J. (1990) A presumed DNA helicase encoded 
by $E R C C-3$ is involved in the human repair disorder xeroderma pigmentosum and Cockayne's syndrome. Cell 62, 777-791

7. Legerski, R. and Peterson, C. Expression cloning of a human DNA repair gene involved in xeroderma pigmentosum group $C$ (1992) Nature $35970-73$

8. Flejter, W.L., Mcdaniel, L.D., Johns D., Friedberg, C. and Schultz, R.A. (1992) Correction of xeroderma pigmentosum complementation group $D$ mutant cell phenotypes by chromosome and gene transfer : involvement of the human ERCC2 DNA repair gene. Proc. Natl. Acad. Sci. USA, 89, 261-265.Itin, P.H., M.D.

9. Troelstra, C., van Gool, A., de Wit, J., Vermeulen, W., Bootsma, D. and Hoeijmakers, J.H.J. (1992) ERCC6, a member of subfamily of putative helicases, is involved in Cockayne's syndrome and preferential repair of active genes Cell $\mathbf{7 1}, 939-953$

10. Strathdee, C.A., Gavish, H., Shannon, W.R. and Buchwald, M. Cloning of cDNAs for Fanconi's anaemia by functional complementation (1992) Nature 356, 763-767

11. Thomson, L.H. (1988) Use of Chinese Hamster Ovary Cell Mutants to Study Human DNA Repair Genes in DNA Repair. A Laboratory Manual of Research Procedures vol. 3 Friedberg E.C. and Hanawalth P.C eds. M. Dekker, Inc. New York, N.Y. 